\title{
Let's Draw: Detecting and Measuring Parkinson's Disease on Smartphones
}

\author{
Elina Kuosmanen \\ Center for Ubiquitous \\ Computing, University of Oulu \\ Oulu, Finland \\ elina.kuosmanen@oulu.fi
}

\author{
Valerii Kan \\ Center for Ubiquitous \\ Computing, University of Oulu \\ Oulu, Finland \\ valerii.kan@oulu.fi
}

\author{
Aku Visuri \\ Center for Ubiquitous \\ Computing, University of Oulu \\ Oulu, Finland \\ aku.visuri@oulu.fi
}

\author{
Simo Hosio \\ Center for Ubiquitous \\ Computing, University of Oulu \\ Oulu, Finland \\ simo.hosio@oulu.fi
}

\author{
Denzil Ferreira \\ Center for Ubiquitous \\ Computing, University of Oulu \\ Oulu, Finland \\ denzil.ferreira@oulu.fi
}

\begin{abstract}
Spiral drawing has been utilized for years as a clinical tool to observe tremors and other abnormal movements in the assessment of different movement disorders. Specifically, in Parkinson's Disease (PD), patients' motor functionalities are measured by various tests, and spiral drawing is one of the proven techniques for assessing the severity of PD motor symptoms. Traditionally, this test is performed on pen and paper, and visually assessed by a clinician. There have been successful efforts for digitizing this test on tablets. Here, we describe a smartphone-based digitized version of the spiral drawing test. Moreover, we introduce a square-shaped drawing to solve an identified challenge of a smaller screen estate: finger occlusion while drawing. Both approaches are evaluated with 8 Parkinson's Disease patients and 6 age-matching control participants. Based on earlier studies and our data, we select suitable motion parameters for quantifying the task. Our results show an observable, statistically difference in performance between users with Parkinson's Disease and the control group in drawing accuracy.
\end{abstract}

\section{Author Keywords}

Parkinson's disease; smartphone; motor assessment; spiral analysis; Archimedean spiral

\section{INTRODUCTION}

Spiral drawing is a commonly used assessment of different movement disorders, but the drawing performance is rarely quantified in practice $[10,21]$. Spiral drawings are usually only visually inspected by clinicians during a patient's visit as they are conducted in pen and paper. Whereas human handwriting has small breaks, a spiral drawing task requires a

\footnotetext{
Permission to make digital or hard copies of all or part of this work for personal or classroom use is granted without fee provided that copies are not made or distributed for profit or commercial advantage and that copies bear this notice and the full citation on the first page. Copyrights for components of this work owned by others than the author(s) must be honored. Abstracting with credit is permitted. To copy otherwise, or republish, to post on servers or to redistribute to lists, requires prior specific permission and/or a fee. Request permissions from permissions@ acm.org.

CHI'20, April 25-30, 2020, Honolulu, HI, USA

(C) 2020 Copyright held by the owner/author(s). Publication rights licensed to ACM. ISBN $\mathrm{xxx}-\mathrm{x}-\mathrm{x} \mathrm{xxx}-\mathrm{xxxxx}-\mathrm{x} / \mathrm{xx} / \mathrm{xx}$
}

DOI: https://doi .org/xx . $\mathrm{xxxx} / \mathrm{xxxxxxx}$. $\mathrm{xxxxxxx}$ continuous movement, and thus they are useful for catching abnormal movements of dystonia, hypokinesia, and tremor [1]. Spiral drawing assessment is useful in tremor evaluation and detection, capturing the frequency, amplitude, and direction of the tremor, and it can be used to distinguish the type of tremor [1]. Tremor can be caused by different conditions, such as Parkinson's Disease (PD), essential tremor, or drug-induced tremor [11]. It has been shown that the spiral drawing time can be used as an objective assessment of upper limb bradykinesia [2]. Spiral drawing can be used also as a general measure for motorics, older age is related to worse performance in spiral drawing on all measures of fine motor skill [12].

There exist attempts for easing and fastening the analysis of the hand-drawn spiral digitally [18], conducting and analyzing the spiral drawing either with a tablet $[24,25,21]$, and smartphones $[9,23]$. Besides spirals, such as lines and circles [6, $25,14]$ have been used in PD symptom evaluation. Louis et al. [18] used spiral drawing in measuring dyskinesia before and after levodopa-medication. Other studies quantified the spiral drawing $[6,23,21]$, distinguished PD patients from healthy individuals $[25,9,14]$ or between PD tremor from essential tremor [23], supplemented and correlated symptoms to UPDRS (Unified Parkinson's Disease Rating Scale [7]) scores.

In this paper, we extend an existing open toolkit for PD symptom assessment called STOP $[15,13]$ by contributing a smartphone-based drawing tool for measuring input anomalies and quantifying the progression of PD. We also extend the preliminary analysis in [16]. Earlier studies show that spiral drawing can capture tremor, bradykinesia and dyskinesia, and the effect of medication on tablets. Smartphones are the desired option over tablets, mainly due to its portability and the recent proliferation of smartphones, including among older adults [3] who represent the largest age-group affected by PD. According to $[4,5,8]$ observations, the severity of PD tremor often diminishes during a goal-oriented voluntary movement. Also based on participants' feedback in [15], this effect might cause tremor to become less evident when a patient is holding a pen. Thus, we rely on one's finger for drawing instead of a stylus to minimize this effect. 
The standard spiral drawing task on a smartphone screen presents a usability challenge. The target drawing line can become occluded by the user's own finger, unable to see the target curve being drawn. Therefore, for this work, we additionally developed a novel square drawing task to validate.

Different shapes were previously evaluated: stars, crosses, and circles [6, 25, 14]; and mental stress such as counting backward, typically increases the tremor amplitude [4, 22]. The square drawing is familiar (i.e., minimizing the effect of finger occlusion) yet allows monitoring four motions juxtaposing hand and finger coordination while requiring the user's attention (i.e., increasing the mental stress). It requires a different mode of motor control due to discrete movements compared to the continuous movement in spiral drawing [26], providing variability to the symptoms' measurements. Based on earlier user experiences with STOP [15], conducting the same task over time feels monotonous, we will add other tasks such as connecting the dots following a sequence to keep the tool engaging.

Our main contributions in this paper are as follow:

1. We revisit previous spiral quantification methods on tablets $[21,24,27,19]$ and introduce them in a smartphone-based solution;

2. We introduce a square-based drawing test, to solve the finger occlusion challenge with spiral drawings for PD;

3. We evaluate our tool with PD patients vs a NonPD agematched healthy control;

4. We provide design insight and implications based on the different metrics used to distinguish between PD and NonPD groups and evaluation feedback.

\section{EXPERIMENTAL SETUP}

\section{Rationale}

Earlier work investigated which parameters or metrics are useful in assessing spiral drawings. Pullman et al. [21] explored a series of benchmarks for spiral quantification. They calculate "smoothness" (i.e., how close the linear transform remains to its mean), "tightness" (i.e., how many turns of the spirals are drawn over its total angular change within a $10-\mathrm{cm}$ square), and "zero-crossing" (i.e., how frequently the linear transform crosses its mean, spiral irregularity). Also, they calculate the "degree of severity," utilizing these parameters, and use drawing speed and pressure. Saunders-Pullman et al. [24] later utilized this technique when studying the spiral drawing performance correlation with UPDRS scores.

Surangsrirat and Thanawattano [27] report that the average and maximum radial error, and crossing rate from the spiral and octagon spiral drawing tasks are ranked higher in PD than their control group. Besides, the average time was significantly longer in PD patients group compared to healthy controls. Unfortunately, it remains unknown the participants' age groups and the number of participants. Memedi et al. [19] calculated the mean drawing speed with Entropy Approximation (also known as ApEn function), skewnes of the drawing speed, the radial and angular velocity with Entropy Approximation, and the total asymmetry (counted from the maximum radiuses of drawing in $x$ - and $y$-plane), and used Principal Component Analysis for feature extraction for classification.

The ApEn function is a measure quantifying the similarity between a chosen window of time series and the next set of windows of the same duration. The approximation requires a regular sampling rate, and to use it. We would have to interpolate our time series data, unavoidably reducing the data entropy. Instead, we use the varying data sampling rate as a measure.

Based on previous studies, we use the following metrics for quantifying spiral analysis:

1. Drawing speed and total time

2. Drawing accuracy (error)

3. Crossing rate (as percentage)

4. Radial and angular velocity

5. Drawing sampling rate and gaps

The parameters 1-4 are used in earlier studies [21, 24, 27, 19], and $5^{\text {th }}$ is introduced by us. We are not able to measure the finger pressure on an Android smartphone. A device with an external stylus (e.g., Samsung Galaxy Note series) is exploited in measuring the stylus point of pressure by earlier studies [24, $21,27]$. For the square analysis, we use a subset of metrics (as $4^{\text {th }}$ does not apply): drawing speed and total time, drawing accuracy, crossing rate and drawing sampling and gaps.

\section{Experimental Apparatus}

We created an Android application with two drawing tasks, one for a spiral and another for a square drawing [16]. Instructions on how to draw the symbol is shown for the user as a picture: the spiral is drawn starting from the center outwards; for the square, the user starts at the bottom left (Figure 1). After the drawing task completes, the application provides drawing performance information such as accuracy and the elapsed time, but this is not shown for the participants in the experiment.

\section{Design and Sampling of Drawing Tasks}

Traditionally, a PD patient draws a spiral with a pencil on a blank paper or in between the successive guidelines of a printed template with a spiral outline. In our tool, to facilitate line drawing error calculations, we bring a model of a spiral to the screen and ask the user to follow the line starting from the center and then outwards. We monitor the position of the finger on the screen, with the maximum frequency allowed by the smartphone screen sensor, and measure the distance to the template. The Archimedean spiral is represented with polar coordinates as

$$
r=a+b \theta
$$

where $r$ is radius, $a$ is a parameter turning the spiral, and $b$ is the distance between successive turnings [27]. We utilize this for calculating the point $(x, y)$ in the template spiral: 


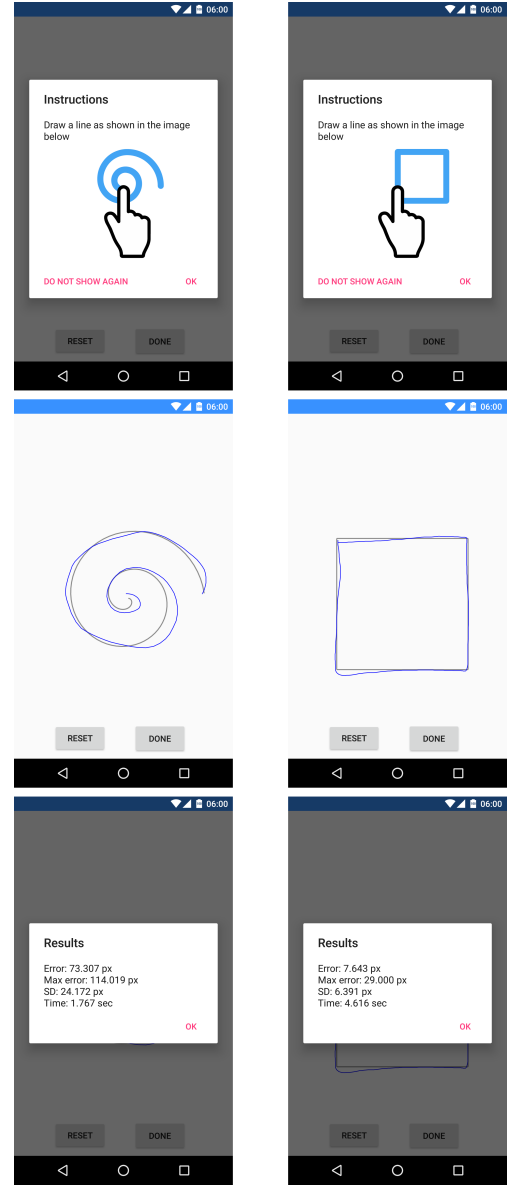

Figure 1. Screen flow of the two drawing tasks - spiral drawing (left) and square drawing (right). From top-down; instructions (top), task (middle), test results (bottom) [16].

$$
\begin{aligned}
& x=r \cos \theta+x_{0}, \\
& y=r \sin \theta+y_{0},
\end{aligned}
$$

where $\left(x_{0}, y_{0}\right)$ are the coordinates of the center of the template spiral [27].

In our application, we incrementally increase the $\theta$ from 0 to $4 \pi$, calculate the coordinates for the template spiral with two loops, and combine the points with a line. For each $\operatorname{dot}\left(x_{i}, y_{i}\right)$ in user drawing, we calculate the radius, in other words the distance from the center, of the template spiral:

$$
r_{i}=\sqrt{\left(y_{i}-y_{0}\right)^{2}+\left(x_{i}-x_{0}\right)^{2}} \text {. }
$$

We can also calculate the angle [27]:

$$
\theta_{i}=\arctan \left(\frac{y_{i}-y_{0}}{x_{i}-x_{0}}\right)
$$

As $\arctan$ gives values only between 0 and $\pi$, we need to keep track on the loops of the spiral, and increase the $\theta_{i}$ accordingly. The angle is used in finding the closest dot in the template

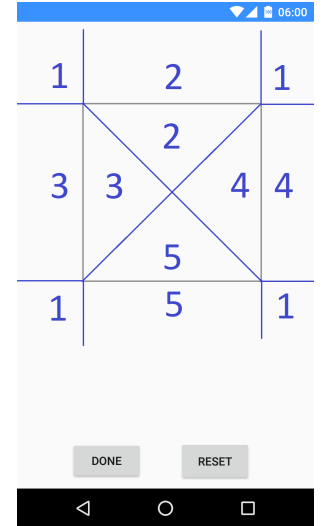

Figure 2. The areas for error calculation when making the square drawing.

spiral:

$$
r_{t}=a+b \theta_{i}
$$

and counting the radial error, similarly as in [27], in each point as:

$$
e_{i}=r_{i}-r_{t}
$$

We sum the absolute values of the errors, and calculate the average error of the drawing as follow:

$$
e r r_{a v}=\frac{1}{n} \sum_{i=1}^{n}\left|e_{i}\right| .
$$

Similarly, we show the outline for the square and ask the user to follow the line. The error calculation for drawing the square is the Euclidean distance to the nearest point in the template square. The screen is divided into areas, as in Fig. 2. For the dots in area 1, the error is distance to the template square corner. From the $2^{\text {nd }}$ and $5^{\text {th }}$ areas, the error is calculated as a distance from template in $y$-plane, and $3^{r d}$ and $4^{\text {th }}$ areas in $x$-plane.

After performing the drawing task, we report to the user the elapsed time, the maximum and average error, with the standard deviation in screen pixel units. Figure 1 exemplifies the task flow presented to the user.

\section{Participants and protocol}

We recruited our 14 participants from a local Parkinson's Association meeting. The study protocol was introduced and all participants signed a consent form agreeing with the data collection and analysis. According to the local Finnish ethics guidelines [20], formal ethical review is not required as there was no foreseeable harm in participation on a drawing task. Before drawing, we asked the participants to fill in a brief paper questionnaire, where we asked their age, if they had PD, the elapsed time since official PD diagnosis, whether they had any other condition affecting their hands' motor skills, and also information concerning their experience with touch screen smartphones.

In total, we recruited 8 participants with PD (4 males, 4 females), and 6 controls without PD ( 2 males, 4 females) with 
matching age groups $\left(M_{P D}=71.5, M_{N o n P D}=72.3\right.$ years of age, $\left.\mathrm{SD}_{P D}=7.0, \mathrm{SD}_{N o n P D}=5.3\right)$. Henceforth these groups are denoted as PD and NonPD.

For our participants, time since PD diagnosis varied between 1-22 years, with an average of 8 years. In the NonPD group, no one reported any condition affecting hands' motor capabilities. We asked if they had conducted a spiral drawing task before, either on paper or digitally. Only one participant (with PD) had earlier experience of spiral drawing tasks. Two PD participants didn't own a touch screen device. All participants were righthanded and used their right hand for drawing.

All participants conducted both drawing tasks (first spiral and then square) three times. All tasks were conducted with the same device provided by us: LGE Nexus 5 (Android 6.0, touchscreen sampling rate of $60 \mathrm{~Hz}$ ) to avoid potential device bias. To debrief, we inquired which hand they use for drawing and whether they are left/right-handed, and asked for their feedback on the drawing tasks. As a reward, we gave the participants a small gift (value $2 €$ ).

We collected the tasks' drawing data as timestamped touch coordinates, and the final drawing screen captures. In total, we captured 24 spirals and 24 squares drawings from participants with PD, and 18 spirals and 18 squares from NonPD, a total of 84 illustrations. We had to exclude three spirals from the data analysis. Participant NONPD1 continued drawing several loops in his first drawing, after which we instructed him when to stop drawing. Participant PD5's touched the screen accidentally with another finger when drawing, causing unintended lines and a high error rate. Participant PD7 drew one extra loop at the beginning of the drawing, and since the error calculation matches the path according to the cumulative angle, it caused a high error rate.

\section{EVALUATION}

During the study, we conducted a brief informal interview with the participants. When asked which PD symptoms affect the hands' motor capabilities, five out of eight reported tremor. Also, stiffness, weakness, sudden movements, and dyskinesia were mentioned. One PD participant told that non-PD related injuries affect as well, in addition to the PD symptoms. We collected users' feedback after the task using an open-ended question: "What are your thoughts regarding this task? Any feedback? If you have performed spiral drawings before, how was this task compared to that?" Generally, the feedback from PD participants was positive. Half described the tasks as "easy", with one instance of "exciting" and "interesting" or that they went "well". Surprisingly, none of the NonPD described the drawing tasks as easy. One referred to them as "surprisingly hard" and another as "challenging". The main rationale was that the touch screen was sensitive, causing errors in the drawings and requiring focusing. Nonetheless, two NonPD participants thought they were "interesting".

\section{1) Drawing speed and total time}

We instructed the participants to follow the line on the screen, without mentioning anything about the time. In each turn, no results are shown to them. Surprisingly, we found no statistical difference between the groups in terms of the total time to draw

\begin{tabular}{lccllll} 
Groups & Min & $Q_{1}$ & Median & Mean & $Q_{3}$ & Max \\
\hline PD spiral & 2.3 & 3.3 & 4.6 & 6.3 & 6.9 & 18.4 \\
NonPD spiral & 2.8 & 3.0 & 3.5 & 3.8 & 4.6 & 5.5 \\
PD square & 3.3 & 3.7 & 5.6 & 6.8 & 6.9 & 19.6 \\
NonPD square & 3.8 & 4.3 & 5.0 & 4.9 & 5.2 & 6.8
\end{tabular}

Table 1. PD and NonPD patients quartile analysis for drawing total time in seconds.

\begin{tabular}{lllllll} 
Groups & Min & $Q_{1}$ & Median & Mean & $Q_{3}$ & Max \\
\hline PD spiral & 0.14 & 0.38 & 0.53 & 0.55 & 0.76 & 1.03 \\
NonPD spiral & 0.49 & 0.62 & 0.67 & 0.69 & 0.80 & 0.89 \\
PD square & 0.17 & 0.39 & 0.49 & 0.51 & 0.70 & 0.81 \\
NonPD square & 0.37 & 0.50 & 0.53 & 0.55 & 0.63 & 0.72
\end{tabular}

Table 2. PD and NonPD patients quartile analysis for average speed in pixels/ms.

the spiral and the square, or the average speed (measured in pixels/millisecond, counted by dividing the drawn line length with the total drawing time), confirmed by a Wilcoxon ranksum test. See Tables 1 and 2. Further investigating the changes in speed (i.e., standard deviation of the drawing speeds, see Fig. 3), we saw that NonPD group has bigger variation in the speed, but again, no statistically significant (Wilcoxon rank-sum test).

\section{2) Drawing accuracy (error)}

Overlaying all the spiral and square drawings per group (Figure 4) with PD on the left and NonPD on the right, we can observe a clear difference in line following adherence.

We find that, on average, NonPD group is $32 \%$ and $24 \%$ more accurate on spiral and square drawings, respectively. The differences between groups in drawing error (Table 3) is statistically significant, and also are the differences between each drawing's maximum error (Table 4), both confirmed by Wilcoxon rank-sum test.

With the spiral drawings, the amount of error in following the template line is visualized by plotting the radius vs. the angle in relation to the spiral line. The optimal trajectory (i.e., the template line) is denoted as the red straight line in Figure 5.

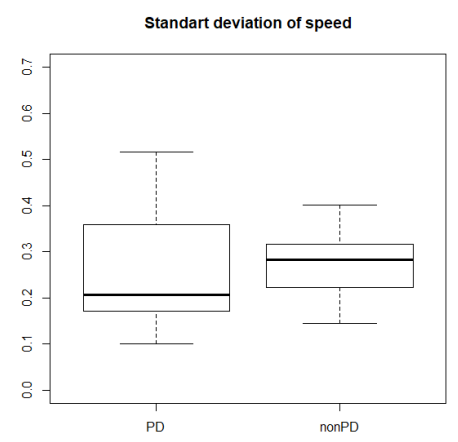

Figure 3. Distribution of standard deviation of speed in spiral drawing. 


\begin{tabular}{lllllll} 
Groups & Min & $Q_{1}$ & Median & Mean & $Q_{3}$ & Max \\
\hline $\begin{array}{l}\text { PD } \\
\text { spiral }\end{array}$ & 16.41 & 21.92 & 34.05 & 31.98 & 39.94 & 46.81 \\
$\begin{array}{l}\text { NonPD } \\
\text { spiral }\end{array}$ & 14.07 & 16.78 & 19.08 & 21.66 & 25.15 & 36.36 \\
$\begin{array}{l}\text { PD } \\
\text { square }\end{array}$ & 17.96 & 24.65 & 28.93 & 29.83 & 34.79 & 42.08 \\
$\begin{array}{l}\text { NonPD } \\
\text { square }\end{array}$ & 15.08 & 19.17 & 22.54 & 22.49 & 25.48 & 28.95
\end{tabular}

Table 3. PD and NonPD patients quartile analysis for average error in pixels.

\begin{tabular}{lllllll} 
Groups & Min & $Q_{1}$ & Median & Mean & $Q_{3}$ & Max \\
\hline $\begin{array}{l}\text { PD } \\
\text { spiral }\end{array}$ & 47.54 & 65.71 & 78.49 & 78.33 & 89.28 & 117.86 \\
$\begin{array}{l}\text { NonPD } \\
\text { spiral }\end{array}$ & 33.00 & 55.79 & 64.48 & 64.24 & 74.63 & 90.30 \\
$\begin{array}{l}\text { PD } \\
\text { square }\end{array}$ & 46.00 & 61.50 & 74.50 & 84.16 & 96.00 & 176.00 \\
$\begin{array}{l}\text { NonPD } \\
\text { square }\end{array}$ & 36.00 & 47.25 & 52.50 & 55.37 & 66.50 & 81.01
\end{tabular}

Table 4. PD and NonPD patients quartile analysis for maximum error in pixels.
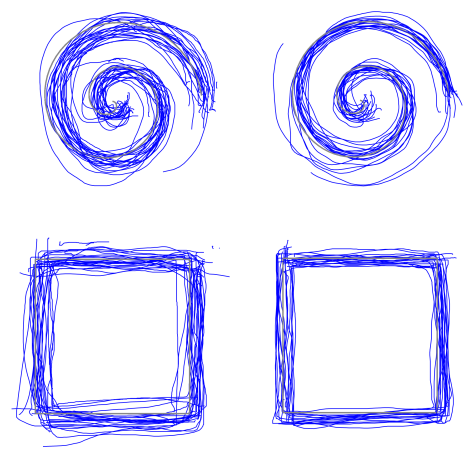

PD

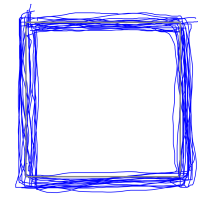

Non-PD

Figure 4. Overlaid of all the participants' drawings for the spiral and square tasks [16].
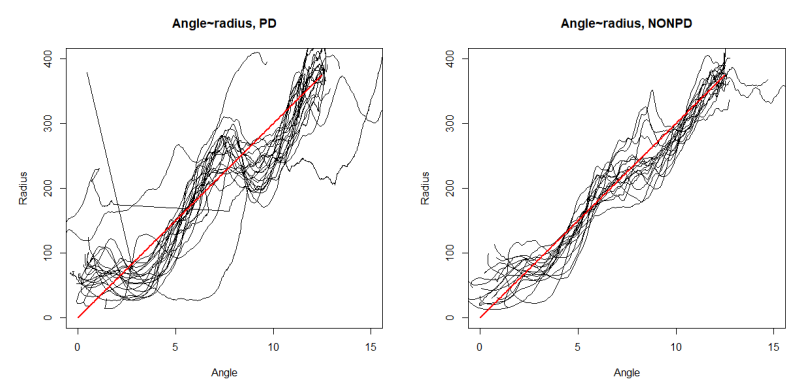

Figure 5. The radius-angle relationship. A perfect spiral (the template) is represented by the red straight line.

\begin{tabular}{lccc} 
Participant & Spiral 1 & Spiral 2 & Spiral 3 \\
\hline NONPD1 & NA & 0.93 & 1.61 \\
NONPD2 & 1.30 & 3.49 & 2.60 \\
NONPD3 & 3.96 & 4.10 & 3.14 \\
NONPD4 & 3.53 & 4.24 & 3.61 \\
NONPD5 & 2.34 & 2.30 & 2.27 \\
NONPD6 & 2.19 & 2.11 & 1.37 \\
PD1 & 3.11 & 2.34 & 3.16 \\
PD2 & 2.23 & 5.07 & 3.59 \\
PD3 & 0.75 & 0.95 & 1.12 \\
PD4 & 1.85 & 2.07 & 1.24 \\
PD5 & 0.12 & 0.79 & 2.78 \\
PD6 & NA & 1.49 & 1.85 \\
PD7 & 0.93 & 1.08 & NA \\
PD9 & 0.75 & 0.00 & 3.68
\end{tabular}

Table 5. The crossing rate in percentage for spiral drawings.

\begin{tabular}{lccc} 
Participant & Square 1 & Square 2 & Square 3 \\
\hline NONPD1 & 1.11 & 0.88 & 1.05 \\
NONPD2 & 1.63 & 1.03 & 1.42 \\
NONPD3 & 1.36 & 2.60 & 2.48 \\
NONPD4 & 1.48 & 3.74 & 3.15 \\
NONPD5 & 1.73 & 0.70 & 0.77 \\
NONPD6 & 3.93 & 2.35 & 1.03 \\
PD1 & 2.51 & 1.97 & 3.47 \\
PD2 & 1.98 & 3.85 & 3.16 \\
PD3 & 1.01 & 0.81 & 1.31 \\
PD4 & 1.97 & 1.05 & 0.67 \\
PD5 & 0.33 & 2.36 & 0.46 \\
PD6 & 0.89 & 2.23 & 3.13 \\
PD7 & 8.62 & 0.58 & 0.60 \\
PD9 & 1.90 & 2.33 & 0.40
\end{tabular}

Table 6. The crossing rate in percentages for square drawings.

Given the sensitivity and drawing sampling rate of a touch screen, NonPD deviate slightly from such a linear relationship (right), but not as much as the PD group (left).

\section{3) Crossing rate}

The crossing rate of a drawing is the number of occurrences where a drawing line crosses the template line, divided by the total number of drawn dots, see Tables 5 and 6 . In spiral drawing, the average of the crossing rate was bigger in NonPD group, $M_{P D}=1.86 \%$. $M_{N o n P D}=2.65 \%$, the difference is statistically significant, proven by Wilcoxon rank-sum test. In the square drawing, the average of crossing rate was slightly larger in PD group $M_{P D}=1.98 \%, M_{N o n P D}=1.80 \%$, but not statistically significantly. These results suggests that a spiral drawing task, is more likely to capture intersections between the template line and the drawn lines.

\section{4) Radial and angular velocity}

We find that the radial and angular velocity of the spiral drawings are, on average, different between groups (radial velocity: $M_{P D}=0.080 \mathrm{pixels} / \mathrm{ms}, M_{N o n P D}=0.096$ pixels $/ \mathrm{ms}$, angular velocity: $M_{P D}=0.003$ radians $/ \mathrm{ms}, M_{N o n P D}=0.004$ radians $/ \mathrm{ms}$ ). 


\begin{tabular}{lcccc} 
Participant & Spiral 1 & Spiral 2 & Spiral 3 & Average \\
\hline NONPD1 & NA & 1.23 & 1.29 & 1.26 \\
NONPD2 & 2.16 & 1.74 & 0.43 & 1.45 \\
NONPD3 & 0.00 & 1.03 & 0.45 & 0.49 \\
NONPD4 & 2.94 & 1.21 & 1.20 & 1.79 \\
NONPD5 & 1.17 & 1.15 & 0.57 & 0.96 \\
NONPD6 & 1.09 & 0.35 & 1.03 & 0.82 \\
PD1 & 0.00 & 3.51 & 1.27 & 1.59 \\
PD2 & 0.89 & 0.72 & 2.99 & 1.54 \\
PD3 & 2.99 & 2.62 & 1.96 & 2.52 \\
PD4 & 1.23 & 1.48 & 1.24 & 1.32 \\
PD5 & 5.21 & NA & 7.26 & 6.23 \\
PD6 & 0.00 & 2.49 & 0.93 & 1.14 \\
PD7 & 0.93 & 1.08 & NA & 1.00 \\
PD9 & 3.24 & 0.54 & 3.68 & 2.49
\end{tabular}

Table 7. The share (\%) of abnormal sampling for spiral drawings.

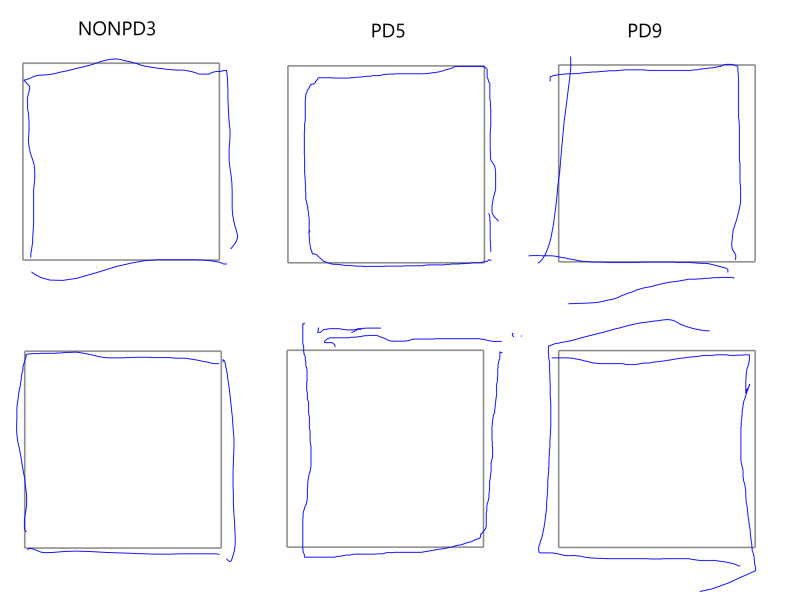

Figure 7. Some participants drew squares in parts. Squares by NONPD3 in first column, PD5 in second column and PD9 in third column.

However, we found no statistical significant difference between the groups, using Wilcoxon rank-sum test. See Fig.6.
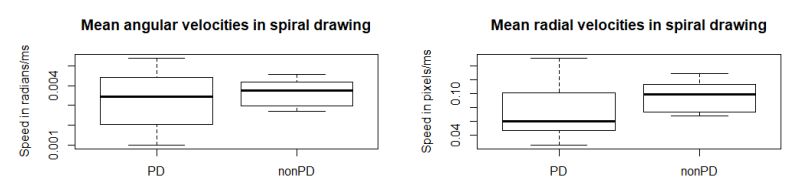

Figure 6. Between groups angular (radians/ms) and radial (pixels/ms) velocities distribution

\section{5) Drawing sampling and gaps}

Parkinson's Disease tremor can affect the motor capabilities of the hand, causing the finger to "jump" on the screen. PD, $16 \%$ of spirals and $16 \%$ of square drawings had gaps (break in the line) when NonPD had $0 \%$ and $11 \%$, respectively. Further observing the NonPD, as well as PD data, we found that some participants would lift their finger upon reaching the square's corners, while the spiral encouraged a continuous line (Figure 7).

\begin{tabular}{lcccc} 
Participant & Square 1 & Square 2 & Square 3 & Average \\
\hline NONPD1 & 4.99 & 3.24 & 2.44 & 3.55 \\
NONPD2 & 3.58 & 2.40 & 3.20 & 3.06 \\
NONPD3 & 6.33 & 3.65 & 7.43 & 5.80 \\
NONPD4 & 3.45 & 2.34 & 4.05 & 3.28 \\
NONPD5 & 4.33 & 4.21 & 3.09 & 3.88 \\
NONPD6 & 0.33 & 1.01 & 0.69 & 0.67 \\
PD1 & 0.50 & 1.97 & 1.49 & 1.32 \\
PD2 & 1.98 & 1.44 & 3.68 & 2.37 \\
PD3 & 2.03 & 1.90 & 1.57 & 1.83 \\
PD4 & 3.28 & 3.85 & 4.68 & 3.94 \\
PD5 & 6.34 & 3.98 & 5.99 & 5.44 \\
PD6 & 3.56 & 1.79 & 1.56 & 2.30 \\
PD7 & 1.85 & 4.68 & 1.79 & 2.77 \\
PD9 & 1.42 & 7.44 & 3.96 & 4.27
\end{tabular}

Table 8. The share (\%) of abnormal sampling for square drawings.

We also found that the drawing sampling rate is not constant during each drawing loop. A normal drawing sampling interval is $16.5 \mathrm{~ms}$ in our device. Thus, we identified abnormal sampling intervals (sampling interval less than $16.5 \mathrm{~ms}$ or above $17 \mathrm{~ms})$. We found more abnormal occurrences in the PD group in spiral drawing: $M_{P D}=2.10 \%, M_{N o n P D}=1.12 \%$, but the opposite for the square drawing: $M_{P D}=2.10 \%, M_{N o n P D}=3.33 \%$, but not statistically significant differencies (Wilcoxon rank-sum test).

As expected, the drawing sampling abnormalities vary per participant, see Tables 3.4, 8. On the intake questionnaire, PD participants PD1, PD3, PD4, PD5, and PD6 reported tremor when asked the symptoms affecting to hand's motorics. PD6 mentioned stiffness, and PD5 and PD9 dyskinesia (i.e., sudden movements). With the visual observation during the drawing we saw that participant PD5 had strong upper limb tremor. PD5 also had high abnormal sampling rate (spiral 6.23\% average, square $5.44 \%$ average).

\section{DISCUSSION}

Our evaluation validates the feasibility for conducting drawing tasks with a smartphone and without stylus for measuring PD symptoms. We are contributing the drawing tasks to be a part of a broader set of tools for longitudinal PD symptom assessment. The toolkit already has mechanisms for medication logging, measuring the motor symptoms (mainly tremor) with a ball-balancing game, and reporting the daily self-evaluated symptom level [15]. With this combination of tools, we can further assess the medication effectiveness and track the PD progression more regularly.

Similar to reported findings in previous studies using exclusively spiral drawings [24, 25, 6, 21, 23], we discovered a difference between the PD group and with the age-matching NonPD control group in the drawing accuracy (i.e., error rate and maximum error). In general, the NonPD group was 32\% and $24 \%$ more accurate in spiral and square drawings, respectively, with a statistically significant difference. We did not find a statistically significant difference in drawing speeds between PD and NonPD groups, even though this was evident 
in previous work [27]. This could simply be because we are using a smaller screen size with less drawing estate. In turn, our tasks were shorter in time.

Digitizing the spiral drawings is not novel, however, we do not require a stylus nor a tablet to perform the assessment. Here, not relying on a stylus, a foreseeable limitation of such drawing is the occlusion of someone's finger over a line. To address such a challenge, we created a new kind of drawing, a square. We chose a square based on the work with straight lines in $[6,25,14]$. A square allows for four instances of a straight line to occur, thus providing a different mode of control. Being a set of straight lines, a square also allows the user to anticipate the trajectory that is expected to be followed by his finger, thus mitigating the finger occlusion limitation that exists with the digital spiral.

In the analysis the crossing rate of NonPD vs PD drawings we find that the difference between PD and NonPD groups were statistically significant for both drawings. Even though the crossing rate of spiral drawing is higher for NonPD, the average error is lower. Hence, the drawings were more compliant to the template, touching the template line more often. On the other hand, the crossing rate in the square drawings is higher for PD, with a higher average error. In other words, NonPD has drawn closer to the template, with straighter lines resulting in fewer line crossings.

In addition, the spiral drawing in a smartphone screen is small if compared with its presentation on a tablet display, which makes it challenging to compare our results with previous deployments and evaluations. Nonetheless, we learned a few design implications along the way. For the drawing accuracy, PD5 had problems with extra finger hitting the screen surface. Being a smaller device to hold, the extra finger causes sharp lines in opposite directions than the drawing finger. Some participants lifted their drawing finger in the middle of task. In the spiral drawing, our algorithm detects when we need to add $\pi$ to the angle due to polar coordinates based on the difference in the angle between successive points. If the finger has been lifted, we might add $\pi$ erroneously causing inaccuracy to calculations.

The participants lifted their finger more while doing the square drawing, as some participants drew in sections at a time. PD9 drew the bottom line twice, and he did a correction closer to the original line (see the rightmost square in the upper row in Fig. 7). The drawing accuracy of a square matches the drawn line linearly to the closest point in the template, accounting all drawn lines for a valid accuracy calculation.

One could argue one way to mitigate the finger lifting issue would be to reset the task when it occurs. However, this might make the task more difficult to complete: $12 \%(10 / 84)$ of the drawings contained a break; $17 \%(8 / 48)$ of the PD group's drawings had breaks, while only 5\% (2/36) of the NonPD group had these issues. Based on our in-situ observations, the tremor affects the motor capabilities of the hand, causing the finger "jumping" in the screen. Resetting the task would likely not be optimal for user experience. Instead, if the participant doesn't draw the whole figure, the missing section should be accounted for as an error. Hence, we must reduce the score accordingly if there are missing parts in the drawing. This approach shall be validated in further studies.

All our participants were experienced adults $\left(M_{P D}=71.5\right.$, $M_{N o n P D}=72.3$ years of age, $\mathrm{SD}_{P D}=7.0, \mathrm{SD}_{N o n P D}=5.3$ ). Our experiments also demonstrate the age effects on fine motor skills for both clinical scores and quantitative spiral-drawing measures, which had already been shown by Hoodendam et al. [12], where his study demonstrated that older age is related to worse fine motor skills by quantitative measures of the Archimedes spiral-drawing test. The age-related motorics weakening may have hidden the PD related symptoms in our study, thus we find a need to extend our tool in the future to assess a baseline for motoric skills to monitor the progression of PD based on individual motoric skills as a starting point.

\section{Future work}

Our study shows, that PD and NonPD groups differ in performance, hence PD symptoms affect one's drawing ability. Our target is to provide a tool for daily PD symptom tracking, but it remains unclear if the drawing tasks we created for a smartphone can be used to measuring the variation of the symptoms and the medication effectiveness, and which symptoms affect the drawing performance in the wild. Our next step is to continue our work with clinicians and explore which symptoms are more likely identified and captured from the drawing tasks. We will combine the drawing data to the data collected by an existing set of tools $[17,13]$. The drawing results can then be compared to the ball-balancing game performance metrics, and these combined with the medication logging so we can estimate the medication effectiveness and lasting effect for informed PD treatments.

\section{Limitations}

Our study is conducted with a small sample size (14 participants), and thus we can only report what we observed within this sample. The amount of data is limited to the controlled experiment we conducted at the Parkinson's Association, but we plan to deploy our toolkit on the Play Store to significantly increase the participants' pool in the future. The age-related motorics weakening may have hidden the PD related symptoms in our study. Nonetheless, our results are indicative that it possible to capture and distinguish between PD and NonPD individuals by means of simple drawing tasks, as we detect statistically significant difference between the groups in various drawing metrics. However, we still need to study if the drawing tasks can be used in the measurement of daily symptom level variation.

\section{CONCLUSION}

In this paper, we present means for quantifying symptoms of Parkinson's disease through a mobile solution that does not require a stylus to work. The work is motivated by the proliferation of the smartphone and its emergence as a handy tool for diagnosing and measuring various health conditions. Not a lot of prior work focuses on PD, specifically. Accessible on an off-the-shelf smartphone, our work allows PD patients to perform the spiral assessment frequently, on a daily or weekly basis, and more importantly, remotely. Our work contributes 
toward a compelling future vision where the measurement data from a rich array of simple-to-use measurement applications on mobile phone are made available to the clinicians of people suffering from PD, thus allowing for informed decisions especially concerning the progress and efficiency of PD medication and any ongoing treatments.

\section{ACKNOWLEDGEMENTS}

This work is partially funded by the Academy of Finland (Grants 313224-STOP, 316253-SENSATE, 320089SENSATE and 318927-6Genesis Flagship), and personal research grants awarded by the Finnish Parkinson Foundation, Tauno Tönning Foundation, Jenny and Antti Wihuri Foundation and Nokia Foundation.

\section{REFERENCES}

[1] Jane Alty, Jeremy Cosgrove, Deborah Thorpe, and Peter Kempster. 2017. How to use pen and paper tasks to aid tremor diagnosis in the clinic. Practical Neurology 17, 6 (2017), 456-463. DOI:

http://dx.doi.org/10.1136/practneurol-2017-001719

[2] Krzysztof Banaszkiewicz, Monika Rudzińska, Sylwia Bukowczan, Andrzej Izworski, and Andrzej Szczudlik. 2009. Spiral drawing time as a measure of bradykinesia. Neurologia i neurochirurgia polska 43, 1 (2009), 16-21. http://europepmc.org/abstract/MED/19353440

[3] Anabela Berenguer, Jorge Goncalves, Simo Hosio, Denzil Ferreira, Theodoros Anagnostopoulos, and Vassilis Kostakos. 2016. Are Smartphones Ubiquitous?: An in-depth survey of smartphone adoption by seniors. IEEE Consumer Electronics Magazine 6, 1 (2016), 104-110.

[4] Kailash P Bhatia, Peter Bain, Nin Bajaj, Rodger J Elble, Mark Hallett, Elan D Louis, Jan Raethjen, Maria Stamelou, Claudia M Testa, Guenther Deuschl, and others. 2018. Consensus Statement on the classification of tremors. from the task force on tremor of the International Parkinson and Movement Disorder Society. Movement Disorders 33, 1 (2018), 75-87.

[5] Carmen Camara, Pedro Isasi, Kevin Warwick, Virginie Ruiz, Tipu Aziz, John Stein, and Eduard Bakštein. 2015. Resting tremor classification and detection in Parkinson's disease patients. Biomedical Signal Processing and Control 16 (2015), 88-97.

[6] Natalia Dounskaia, Arend WA Van Gemmert, Berta C Leis, and George E Stelmach. 2009. Biased wrist and finger coordination in Parkinsonian patients during performance of graphical tasks. Neuropsychologia 47, 12 (2009), 2504-2514.

[7] SRLE Fahn. 1987. Unified Parkinson's disease rating scale. Recent development in Parkinson's disease (1987).

[8] Alexandre Gironell, Berta Pascual-Sedano, Ignacio Aracil, Juan Marín-Lahoz, Javier Pagonabarraga, and Jaime Kulisevsky. 2018. Tremor Types in Parkinson
Disease: A Descriptive Study Using a New

Classification. Parkinson's Disease 2018 (2018).

[9] Ricardo Graça, Rui Sarmento e Castro, and Joao Cevada. 2014. Parkdetect: Early diagnosing parkinson's disease. In Medical Measurements and Applications (MeMeA), 2014 IEEE International Symposium on. IEEE, 1-6.

[10] Christopher W Hess, Annie W Hsu, Qiping Yu, Robert Ortega, and Seth L Pullman. 2014. Increased variability in spiral drawing in patients with functional (psychogenic) tremor. Human movement science 38 (2014), 15-22.

[11] Christopher W Hess and Seth L Pullman. 2012. Tremor: clinical phenomenology and assessment techniques. Tremor and other hyperkinetic movements 2 (2012).

[12] Yoo Young Hoogendam, Fedde van der Lijn, Meike W Vernooij, Albert Hofman, Wiro J Niessen, Aad van der Lugt, M Arfan Ikram, and Jos N van der Geest. 2014. Older age relates to worsening of fine motor skills: a population-based study of middle-aged and elderly persons. Frontiers in aging neuroscience 6 (2014), 259.

[13] Valerii Kan. 2018. STOP: a smartphone-based game for Parkinson's disease medication adherence. (2018).

[14] C Kotsavasiloglou, N Kostikis, Dimitrios Hristu-Varsakelis, and M Arnaoutoglou. 2017. Machine learning-based classification of simple drawing movements in Parkinson's disease. Biomedical Signal Processing and Control 31 (2017).

[15] Elina Kuosmanen, Valerii Kan, Julio Vega, Aku Visuri, Yuuki Nishiyama, Anind K Dey, Simon Harper, and Denzil Ferreira. 2019a. Challenges of Parkinson's Disease: User Experiences with STOP. In Proceedings of the 21st International Conference on Human-Computer Interaction with Mobile Devices and Services. ACM, 22.

[16] Elina Kuosmanen, Valerii Kan, Aku Visuri, Assam Boudjelthia, Lokmane Krizou, and Denzil Ferreira. 2019b. Measuring Parkinson's disease motor symptoms with smartphone-based drawing tasks. In Proceedings of the 2019 ACM International Joint Conference on Pervasive and Ubiquitous Computing and Proceedings of the 2019 ACM International Symposium on Wearable Computers. ACM, 1182-1185.

[17] Elina Kuosmanen, Valerii Kan, Aku Visuri, Julio Vega, Yuuki Nishiyama, Anind K Dey, Simon Harper, and Denzil Ferreira. 2018. Mobile-based Monitoring of Parkinson's Disease. In Proceedings of the 17th International Conference on Mobile and Ubiquitous Multimedia. ACM, 441-448.

[18] Elan D Louis, Qianhua Zhao, Haijiao Meng, and Ding Ding. 2012. Screening for action tremor in epidemiological field surveys: assessing the reliability of a semi-quantitative, visual, template-based scale for rating hand-drawn spirals. Tremor and Other Hyperkinetic Movements 2 (2012). 
[19] Mevludin Memedi, Aleksander Sadikov, Vida Groznik, Jure Žabkar, Martin Možina, Filip Bergquist, Anders Johansson, Dietrich Haubenberger, and Dag Nyholm. 2015. Automatic spiral analysis for objective assessment of motor symptoms in Parkinson's disease. Sensors 15, 9 (2015), 23727-23744.

[20] National Advisory Board on Research Ethics. 2009. Ethical principles of research in the humanities and social and behavioural sciences and proposals for ethical review. (2009). Retrieved December 10, 2019 from http://www.tenk.fi/sites/tenk.fi/files/ ethicalprinciples.pdf

[21] Seth L Pullman. 1998. Spiral analysis: a new technique for measuring tremor with a digitizing tablet. Movement Disorders 13, S3 (1998), 85-89.

[22] Jan Raethjen, Kristina Austermann, Karsten Witt, Kirsten E Zeuner, Frank Papengut, and Günther Deuschl. 2008. Provocation of Parkinsonian tremor. Movement disorders: official journal of the Movement Disorder Society 23, 7 (2008), 1019-1023.

[23] Aleksander Sadikov, Vida Groznik, Jure Žabkar, Martin Možina, Dejan Georgiev, Zvezdan Pirtošek, and Ivan Bratko. 2014. Parkinson Check smart phone app. In
Proceedings of the Twenty-first European Conference on Artificial Intelligence. IOS Press, 1213-1214.

[24] Rachel Saunders-Pullman, Carol Derby, Kaili Stanley, Alicia Floyd, Susan Bressman, Richard B Lipton, Amanda Deligtisch, Lawrence Severt, Qiping Yu, Mónica Kurtis, and others. 2008. Validity of spiral analysis in early Parkinson's disease. Movement disorders: official journal of the Movement Disorder Society 23, 4 (2008), 531-537.

[25] Esther J Smits, Antti J Tolonen, Luc Cluitmans, Mark van Gils, Bernard A Conway, Rutger C Zietsma, Klaus L Leenders, and Natasha M Maurits. 2014. Standardized handwriting to assess bradykinesia, micrographia and tremor in Parkinson's disease. PloS one 9, 5 (2014), e97614.

[26] Rebecca MC Spencer, Howard N Zelaznik, Jörn Diedrichsen, and Richard B Ivry. 2003. Disrupted timing of discontinuous but not continuous movements by cerebellar lesions. science 300, 5624 (2003), 1437-1439.

[27] Decho Surangsrirat and Chusak Thanawattano. 2012. Android application for spiral analysis in Parkinson's Disease. In Southeastcon, 2012 Proceedings of IEEE. IEEE, 1-6. 